\title{
A NOTE ON THE NUMERICAL INTEGRATION OF A CLASS OF NON-LINEAR HYPERBOLIC EQUATIONS*
}

By R. BELLMAN (The RAND Corp., Santa Monica, Calif.) AND I. CHERRY AND G. M. WING (Los Alamos Scientific Laboratory, University of California, Los Alamos, N. M.)

1. Introduction. Since the equations of hydrodynamics are non-linear, a computational solution of these equations usually involves a numerical integration. This numerical integration is particularly difficult to perform in the presence of discontinuities of the solution called "shocks." Although there are a number of devices available for obtaining the solution in the neighborhood of the shock, notably by ron Neumann, Richtmeyer and Lax, these have the unfortunate property of altering the behavior of the solution in other regions. If conventional finite difference schemes are employed, instabilities arise in a number of cases.

In this paper we propose a new method of numerical integration which seems well suited to non-linear hyperbolic equations of the form

$$
u_{t}=g(u) u_{x}+h(u, x, t), \quad u(x, 0)=f(x) .
$$

To illustrate the method, we consider the pseudo-hydrodynamic equation

$$
u_{t}=-u u_{x}, \quad u(x, 0)=f(x),
$$

which exhibits the shock phenomenon and which possesses the great merit of an analytic solution. We can thus compare the analytic solution with that obtained from the application of our numerical technique.

2. The equation $u_{t}=-u u_{x}$. The equation appearing in (1.2) has the solution

$$
u(x, t)=f[x-u(x, t) t] .
$$

Since

$$
u_{x}(x, t)=\frac{f^{\prime}(E)}{1+t f^{\prime}(E)}, \quad E=x-u(x, t) t,
$$

it is clear that $u_{x}$ becomes infinite whenever $1+t f^{\prime}(E)=0$. This is the shock phenomenon. Numerical integration is difficult in the neighborhood of this shock.

In place of the usual finite difference method based upon a fixed $(x, t)$-grid, consider the following scheme:

$$
u(x, t+\Delta)=u[x-u(x, t) \Delta, t], \quad u(x, 0)=f(x),
$$

where $t$ assumes the values $0, \Delta, 2 \Delta, \cdots$. Expansion of the two sides in powers of $\Delta$ reveals that (3) is equivalent to (1.2) to first order terms in $\Delta$.

The method does not involve the strict notion of an $(x, t)$ grid, since the $x-u(x, t) \Delta$ values will not be distributed in any regular fashion. In integrating the equation, a fixed $x$-grid is used, $x=\cdots,-2 \delta,-\delta, 0, \delta, 2 \delta, \cdots$. When the values $u(n \delta, t)$ are known

${ }^{*}$ Received April 23, 1957; revised manuscript received June 18, 1957. Work done under the auspices of the U. S. Atomic Energy Commission. 
for fixed $t$ the quantities

$$
u[n \delta-u(n \delta, t) \Delta, t]=u(n \delta, t+\Delta)
$$

are found by interpolation in the $x$ direction. There is no constraint on the relation between $\delta$ and $\Delta$, and $\delta$ may be chosen as small as desired, subject to space limitations on the digital computer and the cost of computing time.

One important feature of the recurrence scheme in (3) above is that uniform boundedness of $u(x, t)$ over $x$ for $t=k \Delta$ is automatically passed on to $u(x, t)$ at $t=(k+1) \Delta$.

3. An example. We have integrated (2.3) using for the starting condition

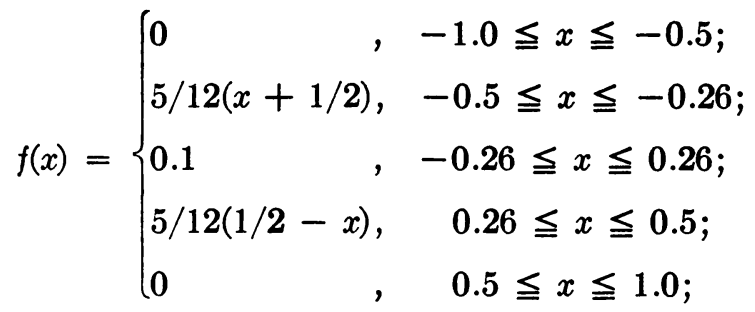

$$
\begin{aligned}
& f(x)=f(x+2), \quad-\infty<x<\infty .
\end{aligned}
$$

We have used six-point Lagrangian interpolation, $x$ points at intervals of $\delta=0.02$, and various values of $\Delta$. Some results are shown in the table. It should be noted that even with relatively large $\Delta$ the discontinuity in $u$ that takes place at $t=2.4$ is handled quite well. Other starting conditions have also proved successful.

4. Discussion. Since the method consists of integrating along the characteristic, good results are to be expected. However, we have also integrated equations of the form

$$
\begin{aligned}
u_{t} & =g(u) u_{x}+h(u, x, t) \\
u(x, 0) & =f(x), \quad-\infty<x<\infty,
\end{aligned}
$$

using the difference scheme

$$
\begin{aligned}
u(x, t+\Delta)= & u(x-g[u(x, t)] \Delta, t) \\
& +h[u(x, t), x, t] \Delta, \\
u(x, 0)= & f(x), \quad-\infty<x<\infty,
\end{aligned}
$$

where the characteristic curves are not simply $x-g(u) t=k$, and have obtained excellent results.

5. Extension. The method is applicable to the integration of the system

$$
\begin{aligned}
u_{t} & =G(u, v, x, y) u_{x}+H(u, v, x, y) u_{y}+g(u, v, x, y), \\
v_{t} & =M(u, v, x, y) u_{x}+N(u, v, x, y) v_{v}+h(u, v, x, y) .
\end{aligned}
$$

The corresponding difference scheme is

$$
\begin{aligned}
& u(x, y, t+\Delta)=u\{x+G \Delta, y+H \Delta, t\}+g \Delta \\
& v(x, y, t+\Delta)=v\{x+M \Delta, y+N \Delta, t\}+h \Delta .
\end{aligned}
$$

It is readily verified that (5.2) is equivalent to (5.1) to terms of order $\Delta$. The inte- 
gration proceeds as in the previous case, a grid in the $(x, y)$ plane being chosen and interpolation among these grid points being used to advance the integration in time.

However, attempts at extending the integration method in an obvious way to general systems of hyperbolic equations in one space variable have not been successful. While the method presented here is marginally stable, the attempted generalizations have suffered from instabilities.

TABLE 1

Values of $u(x, t)$ at $t=2.4$.

\begin{tabular}{l|l|l|l|l|l}
\hline$x$ & \multirow{2}{*}{ Exact $u$} & \multicolumn{4}{|c}{ Computed $u$} \\
\cline { 2 - 6 } & & $\Delta=0.02$ & $\Delta=0.10$ & $\Delta=0.20$ & $\Delta=0.30$ \\
\hline-.48 & 0.004167 & 0.003713 & 0.003657 & 0.003584 & 0.003508 \\
-.40 & 0.0208 & 0.020805 & 0.02056 & 0.02024 & 0.01991 \\
-.32 & 0.0375 & 0.03738 & 0.03694 & 0.03637 & 0.03576 \\
-.24 & 0.0542 & 0.05399 & 0.05336 & 0.05253 & 0.05165 \\
-.16 & 0.0708 & 0.07063 & 0.06981 & 0.06869 & 0.06745 \\
-.08 & 0.0875 & 0.08748 & 0.08621 & 0.08484 & 0.08255 \\
.00 & 0.1000 & 0.10022 & 0.10034 & 0.10000 & 0.10072 \\
+.08 & 0.1000 & 0.09994 & 0.10000 & 0.10000 & 0.10002 \\
+.16 & 0.1000 & 0.10000 & 0.10000 & 0.10000 & 0.10000 \\
+.24 & 0.1000 & 0.10000 & 0.10000 & 0.10000 & 0.10000 \\
+.32 & 0.1000 & 0.10002 & 0.10000 & 0.10000 & 0.10000 \\
+.40 & 0.1000 & 0.10028 & 0.10008 & 0.10000 & 0.10009 \\
+.44 & 0.1000 & 0.09924 & 0.09952 & 0.10000 & 0.10111 \\
+.46 & 0.1000 & 0.09785 & 0.10351 & 0.09884 & 0.08966 \\
+.48 & 0.1000 & 0.12222 & 0.08834 & 0.06096 & 0.04563 \\
+.50 & - & 0.00000 & 0.00000 & 0.00000 & 0.00000 \\
+.52 & 0.0000 & 0.00000 & 0.00000 & 0.00000 & 0.00000 \\
+.54 & 0.0000 & 0.00000 & 0.00000 & 0.00000 & 0.00000 \\
+.56 & 0.0000 & 0.00000 & 0.00000 & 0.00000 & 0.00000 \\
\hline
\end{tabular}

\section{ON THE EQUIDISTRIBUTION OF PSEUDO-RANDOM NUMBERS*}

\section{By J. N. FRANKLIN (California Institute of Technology)}

1. Introduction. In the paper [1] J. Moshman discusses a method for generating pseudo-random numbers. As Moshman points out, his method is an adaptation to decimal computers of a process used on a binary computer by J. Todd and O. Taussky Todd [2]. Another adaptation to decimal computers was made by M. Juncosa [3]. The method is used widely for computations in nuclear physics. For example, the method is used by E. Leshan [4] to study the transport of neutrons.

In this method one chooses an initial number $x_{0}$ and a multiplier $N$. Each of these numbers is represented in a digital computer by a word with a fixed number of digits, say $s$ digits. The product $N x_{0}$ contains $2 s$ digits, of which the $s$ least significant digits

*Received May 9, 1957. 